\title{
COBALANCING NUMBERS AND COBALANCERS
}

\author{
G. K. PANDA AND P. K. RAY \\ Received 11 May 2004 and in revised form 16 February 2005
}

Cobalancing numbers and cobalancers are defined and introduced. Many properties of cobalancing numbers are explored. A link between the Pythagorean triplets and the cobalancing numbers is also established.

\section{Introduction}

Recently, Behera and Panda [1] introduced balancing numbers $n \in \mathbb{Z}^{+}$as solutions of the equation

$$
1+2+\cdots+(n-1)=(n+1)+(n+2)+\cdots+(n+r),
$$

calling $r \in \mathbb{Z}^{+}$the balancer corresponding to the balancing number $n$. The numbers 6 , 35 , and 204 are examples of balancing numbers with balancers 2,14 , and 84 , respectively. Behera and Panda [1] also proved that a positive integer $n$ is a balancing number if and only if $n^{2}$ is a triangular number, that is, $8 n^{2}+1$ is a perfect square. Though the definition of balancing numbers suggests that no balancing number should be less than 2, in [1], 1 is accepted as a balancing number being the positive square root of the square triangular number 1.

In $[4,5]$, Subramaniam has explored some interesting properties of square triangular numbers. In a latter paper [6], he introduced the concept of almost square triangular numbers (triangular numbers that differ from a square by unity) and established links with the square triangular numbers. In this paper, we introduce cobalancing numbers and see that they are very closely associated with balancing numbers and also with triangular numbers which are products of two consecutive natural numbers. Observe that a number, which can be expressed as a product of two consecutive natural numbers, is almost equal to the arithmetic mean of squares of two consecutive natural numbers, that is, $n(n+1) \approx$ $\left[n^{2}+(n+1)^{2}\right] / 2$. In what follows, we introduce the cobalancing numbers in a way similar to the balancing numbers.

By slightly modifying (1.1), we call $n \in \mathbb{Z}^{+}$a cobalancing number if

$$
1+2+\cdots+n=(n+1)+(n+2)+\cdots+(n+r)
$$


for some $r \in \mathbb{Z}^{+}$. Here, we call $r$ the cobalancer corresponding to the cobalancing number $n$.

The first three cobalancing numbers are 2, 14, and 84 with cobalancers 1,6 , and 35 , respectively.

It is clear from (1.2) that $n$ is a cobalancing number with cobalancer $r$ if and only if

$$
n(n+1)=\frac{(n+r)(n+r+1)}{2}
$$

which when solved for $r$ gives

$$
r=\frac{-(2 n+1)+\sqrt{8 n^{2}+8 n+1}}{2}
$$

It follows from (1.4) that $n$ is a cobalancing number if and only if $8 n^{2}+8 n+1$ is a perfect square, that is, $n(n+1)$ is a triangular number. Since $8 \times 0^{2}+8 \times 0+1=1$ is a perfect square, we accept 0 as a cobalancing number, just like Behera and Panda [1] accepted 1 as a balancing number, though, by definition, a cobalancing number should be greater than 1 .

From the above discussion, it is clear that if $n$ is a cobalancing number, then both $n(n+1)$ and $n(n+1) / 2$ are triangular numbers. Thus, our search for cobalancing number is confined to the pronic triangular numbers, that is, triangular numbers that are also pronic numbers. It is worth mentioning here that a positive integer is called a pronic number if it is expressible in the form $n(n+1)$ for some positive integer $n$. Since $n<$ $\sqrt{n(n+1)}<n+1$, it follows that if $T$ is a pronic triangular number, then $[\sqrt{T}]$ must be a cobalancing number, where $[\cdot]$ denotes the greatest integer function. For example, $T=6$ is a pronic triangular number, and therefore $[\sqrt{ } 6]=2$ is a cobalancing number.

\section{Some functions of cobalancing numbers}

In this section, we introduce some functions of cobalancing numbers that also generate cobalancing numbers. For any two cobalancing numbers $x$ and $y$, we consider the following functions:

$$
\begin{aligned}
f(x) & =3 x+\sqrt{8 x^{2}+8 x+1}+1 \\
g(x) & =17 x+6 \sqrt{8 x^{2}+8 x+1}+8 \\
h(x) & =8 x^{2}+8 x+1+(2 x+1) \sqrt{8 x^{2}+8 x+1}+1 \\
t(x, y)= & \frac{1}{2}\left[2(2 x+1)(2 y+1)+(2 x+1) \sqrt{8 y^{2}+8 y+1}\right. \\
& \left.+(2 y+1) \sqrt{8 x^{2}+8 x+1}+\sqrt{8 x^{2}+8 x+1} \sqrt{8 y^{2}+8 y+1}-1\right] .
\end{aligned}
$$

We first prove that the above functions always generate cobalancing numbers.

Theorem 2.1. For any two cobalancing numbers $x$ and $y, f(x), g(x), h(x)$, and $t(x, y)$ are all cobalancing numbers. 
Proof. Suppose that $u=f(x)$. Then $x<u$ and

$$
x=3 u-\sqrt{8 u^{2}+8 u+1}+1 .
$$

Since $x$ and $u$ are nonnegative integers, $8 u^{2}+8 u+1$ must be a perfect square, and hence $u$ is a cobalancing number.

Since $f(f(x))=g(x)$, it follows that $g(x)$ is also a cobalancing number.

We can also directly verify that $8 h^{2}(x)+8 h(x)+1$ and $8 t^{2}(x, y)+8 t(x, y)+1$ are perfect squares so that $h(x)$ and $t(x, y)$ are cobalancing numbers. But these verifications would involve lengthy algebra. To avoid algebraic complications, we provide relatively easy proofs of these results in Section 6 using Theorem 6.1.

Next we show that for any cobalancing number $x, f(x)$ is not merely a cobalancing number, but it is the cobalancing number next to $x$.

THeOREM 2.2. If $x$ is any cobalancing number, then the cobalancing number next to $x$ is $f(x)=3 x+\sqrt{8 x^{2}+8 x+1}+1$ and consequently, the previous one is $\tilde{f}(x)=3 x-\sqrt{8 x^{2}+8 x+1}$ +1 .

Proof. The proof of the fact that $f(x)=3 x+\sqrt{8 x^{2}+8 x+1}+1$ is the cobalancing number next to $x$ is exactly same as the proof of Theorem 3.1 of [1], and hence it is omitted. Since $f(\tilde{f}(x))=x$, it follows that $\tilde{f}(x)$ is the largest cobalancing number less than $x$.

\section{Recurrence relations for cobalancing numbers}

For $n=1,2, \ldots$, let $b_{n}$ be the $n$th cobalancing number. We set $b_{1}=0$. The next two cobalancing numbers are $b_{2}=2$ and $b_{3}=14$.

Behera and Panda [1], while accepting 1 as a balancing number, have set $B_{0}=1, B_{1}=6$, and so on, using the symbol $B_{n}$ for the $n$th balancing number. To standardize the notation at par with Fibonacci numbers, we relabel the balancing numbers by setting $B_{1}=1, B_{2}=$ 6 , and so on.

Theorem 2.2 suggests that

$$
\begin{aligned}
& b_{n+1}=3 b_{n}+\sqrt{8 b_{n}^{2}+8 b_{n}+1}+1, \\
& b_{n-1}=3 b_{n}-\sqrt{8 b_{n}^{2}+8 b_{n}+1}+1 .
\end{aligned}
$$

Adding the last two equations, we arrive at the conclusion that the cobalancing numbers obey the second-order linear recurrence relation

$$
b_{n+1}=6 b_{n}-b_{n-1}+2 .
$$

An immediate consequence of (3.2) is the following theorem.

TheOREM 3.1. Every cobalancing number is even.

Proof. The proof is based on mathematical induction. The first two cobalancing numbers $b_{1}=0$ and $b_{2}=2$ are even. Assume that $b_{n}$ is even for $n \leq k$. Using (3.2), one can easily see that $b_{k+1}$ is also even. 
Using the recurrence relation (3.2), we can derive some other interesting relations among the cobalancing numbers.

Theorem 3.2. (a) $\left(b_{n}-1\right)^{2}=1+b_{n-1} b_{n+1}$,

(b) for $n>k \geq 2$,

$$
b_{n}=b_{k}+B_{k} b_{n-k+1}-B_{k-1} b_{n-k}
$$

(c) $b_{2 n}=B_{n} b_{n+1}-b_{n}\left(B_{n-1}-1\right)$,

(d) $b_{2 n+1}=\left(B_{n+1}+1\right) b_{n+1}-B_{n} b_{n}$.

Proof. From (3.2), we have

$$
\frac{b_{n+1}+b_{n-1}-2}{b_{n}}=6 \text {. }
$$

Replacing $n$ by $n-1$, we obtain

$$
\frac{b_{n}+b_{n-2}-2}{b_{n-1}}=6 \text {, }
$$

which implies that

$$
\frac{b_{n+1}+b_{n-1}-2}{b_{n}}=\frac{b_{n}+b_{n-2}-2}{b_{n-1}},
$$

which when rearranged gives

$$
\left(b_{n}-1\right)^{2}-b_{n-1} b_{n+1}=\left(b_{n-1}-1\right)^{2}-b_{n-2} b_{n} .
$$

Now, iterating recursively, we obtain

$$
\left(b_{n}-1\right)^{2}-b_{n-1} b_{n+1}=\left(b_{2}-1\right)^{2}-b_{1} b_{3}=(2-1)^{2}-0 \times 14=1 \text {, }
$$

from which (a) follows.

The proof of (b) needs an important link between balancing numbers and cobalancing numbers, which is to be established in the next section after Theorem 4.1. Until then, we postpone the proof of (b).

The proof of (c) follows from (b) by replacing $n$ by $2 n$ and $k$ by $n$. Similarly, the proof of (d) follows from (b) by replacing $n$ by $2 n+1$ and $k$ by $n+1$.

\section{Generating function for cobalancing numbers}

In Section 3, we developed the recurrence relation $b_{n+1}=6 b_{n}-b_{n-1}+2$ for cobalancing numbers. Using this recurrence relation, we first obtain the generating function for 
cobalancing numbers and then establish a very interesting link between balancing numbers and cobalancing numbers.

Recall that the ordinary generating function [3, page 29] for a sequence $\left\{x_{n}\right\}_{n=0}^{\infty}$ of real numbers is defined as

$$
g(s)=\sum_{n=0}^{\infty} x_{n} s^{n}
$$

From [1], we know that the generating function for the sequence of balancing numbers $\left\{B_{n}\right\}_{n=0}^{\infty}$ is

$$
g(s)=\frac{1}{1-6 s+s^{2}}
$$

But in accordance with the new convention as suggested in the previous section, one can easily see that the generating function for the sequence of balancing numbers $\left\{B_{n}\right\}_{n=1}^{\infty}$ takes the form

$$
g(s)=\frac{s}{1-6 s+s^{2}} .
$$

THEOREM 4.1. The generating function for the sequence of cobalancing numbers $\left\{b_{n}\right\}_{n=1}^{\infty}$ is

$$
f(s)=\frac{2 s^{2}}{(1-s)\left(1-6 s+s^{2}\right)},
$$

and consequently for $n \geq 2$,

$$
b_{n}=2\left(B_{1}+B_{2}+\cdots+B_{n-1}\right) .
$$

Proof. From (3.2), for $n=1,2, \ldots$, we have $b_{n+2}-6 b_{n+1}+b_{n}=2$. Multiplying both sides by $s^{n+2}$ and summing over $n=1$ to $n=\infty$, we obtain

$$
\sum_{n=1}^{\infty} b_{n+2} s^{n+2}-6 s \sum_{n=1}^{\infty} b_{n+1} s^{n+1}+s^{2} \sum_{n=1}^{\infty} b_{n} s^{n}=2 s^{2} \sum_{n=1}^{\infty} s^{n},
$$

which in terms of $f(s)$ can be expressed as

$$
\left(f(s)-2 s^{2}\right)-6 s f(s)+s^{2} f(s)=\frac{2 s^{3}}{1-s} .
$$

Thus,

$$
\begin{aligned}
f(s) & =\frac{2 s^{2}}{(1-s)\left(1-6 s+s^{2}\right)}=\frac{2 s}{1-s} \cdot \frac{s}{1-6 s+s^{2}} \\
& =\frac{2 s}{1-s} \cdot g(s)=2\left(s+s^{2}+\cdots\right) g(s) .
\end{aligned}
$$

Now for $n \geq 2$, the coefficient of $s^{n}$ in $f(s)$ can be obtained by collecting the coefficient of $s^{r}$ from $g(s)$ and the coefficient of $s^{n-r}$ from $2\left(s+s^{2}+\cdots\right)$ for $r=1,2, \ldots, n-1$. While 
the coefficient of $s^{r}$ in $g(s)$ is $B_{r}$, the coefficient of $s^{n-r}$ in $2\left(s+s^{2}+\cdots\right)$ is 2 . Hence,

$$
b_{n}=2\left(B_{1}+B_{2}+\cdots+B_{n-1}\right) .
$$

This completes the proof.

The following corollary and Theorem 3.1 are direct consequences of Theorem 4.1.

Corollary 4.2. $B_{n}=\left(b_{n+1}-b_{n}\right) / 2$.

We are now in a position to prove Theorem 3.2(b).

Proof of Theorem 3.2(b). The proof is based on induction on $k$. It is easy to see that the assertion is true for $n>k=2$. Assume that the assertion is true for $n>r \geq k \geq 2$, that is,

$$
b_{n}=b_{r}+B_{r} b_{n-r+1}-B_{r-1} b_{n-r} .
$$

From [1], we know that the balancing numbers obey the recurrence relation

$$
B_{n+1}=6 B_{n}-B_{n-1} .
$$

Applying this relation, (3.2), (4.10), and Corollary 4.2 to (4.10), we obtain

$$
\begin{aligned}
b_{r+1} & +B_{r+1} b_{n-r}-B_{r} b_{n-r-1} \\
& =b_{r+1}+\left(6 B_{r}-B_{r-1}\right) b_{n-r}-B_{r}\left(6 b_{n-r}-b_{n-r+1}+2\right) \\
& =b_{r+1}-2 B_{r}+B_{r} b_{n-r+1}-B_{r-1} b_{n-r} \\
& =b_{r}+B_{r} b_{n-r+1}-B_{r-1} b_{n-r}=b_{n} .
\end{aligned}
$$

Thus, the assertion is also true for $k=r+1$. This completes the proof of Theorem 3.2(b).

\section{Binet form for cobalancing numbers}

From Section 4, we know that the cobalancing numbers satisfy the recurrence relation

$$
b_{n+1}=6 b_{n}-b_{n-1}+2
$$

which is a second-order linear nonhomogeneous difference equation with constant coefficients. Substituting $c_{n}=b_{n}+1 / 2$, we see that $c_{n}$ obey the recurrence relation

$$
c_{n+1}=6 c_{n}-c_{n-1}
$$

which is homogeneous. The general solution of this equation is

$$
c_{n}=A \lambda_{1}^{n}+B \lambda_{2}^{n}
$$

where $\lambda_{1}=3+\sqrt{8}$ and $\lambda_{2}=3-\sqrt{8}$ are the two roots of the auxiliary equation

$$
\lambda^{2}-6 \lambda+1=0
$$


Substituting $c_{1}=1 / 2$ and $c_{2}=5 / 2$ into (5.3), we obtain

$$
A=\frac{1}{\sqrt{\lambda_{1}}\left(\lambda_{1}-\lambda_{2}\right)}, \quad B=\frac{1}{\sqrt{\lambda_{2}}\left(\lambda_{1}-\lambda_{2}\right)},
$$

where $\sqrt{\lambda_{1}}=1+\sqrt{2}$ and $\sqrt{\lambda_{2}}=1-\sqrt{2}$. Thus,

$$
c_{n}=A \lambda_{1}^{n}+B \lambda_{2}^{n}=\frac{\lambda_{1}^{n-1 / 2}-\lambda_{2}^{n-1 / 2}}{\lambda_{1}-\lambda_{2}}, \quad n=1,2, \ldots,
$$

which implies that

$$
b_{n}=\frac{\lambda_{1}^{n-1 / 2}-\lambda_{2}^{n-1 / 2}}{\lambda_{1}-\lambda_{2}}-\frac{1}{2}, \quad n=1,2, \ldots .
$$

The above discussion proves the following theorem.

THEOREM 5.1. If $b_{n}$ is the $n$th cobalancing number, then its Binet form is

$$
b_{n}=\frac{\lambda_{1}^{n-1 / 2}-\lambda_{2}^{n-1 / 2}}{\lambda_{1}-\lambda_{2}}-\frac{1}{2}, \quad n=1,2, \ldots,
$$

where $\lambda_{1}=3+\sqrt{8}, \lambda_{2}=3-\sqrt{8}, \lambda_{1}^{1 / 2}=1+\sqrt{2}$, and $\lambda_{2}^{1 / 2}=1-\sqrt{2}$.

\section{Relations among balancing numbers, cobalancing numbers, balancers, and cobalancers}

Let $B$ be any balancing number with balancer $R$, and $b$ any cobalancing number with cobalancer $r$. Then by definition, the pairs $(B, R)$ and $(b, r)$ satisfy, respectively,

$$
\begin{gathered}
1+2+\cdots+(B-1)=(B+1)+(B+2)+\cdots+(B+R), \\
1+2+\cdots+b=(b+1)+(b+2)+\cdots+(b+r) .
\end{gathered}
$$

Solving (6.1) for $B$ and (6.2) for $b$, we find

$$
\begin{gathered}
B=\frac{(2 R+1)+\sqrt{8 R^{2}+8 R+1}}{2}, \\
b=\frac{(2 r-1)+\sqrt{8 r^{2}+1}}{2} .
\end{gathered}
$$

We infer from (6.3) that if $R$ is a balancer, then $8 R^{2}+8 R+1$ is a perfect square and from (6.4), we conclude that if $r$ is a cobalancer, then $8 r^{2}+1$ is a perfect square.

The above discussion proves the following theorem.

THEOREM 6.1. Every balancer is a cobalancing number and every cobalancer is a balancing number.

For $n=1,2, \ldots$, as usual, let $B_{n}$ be the $n$th balancing number and $b_{n}$ the $n$th cobalancing number. We also denote by $R_{n}$ the balancer corresponding to $B_{n}$, and $r_{n}$ the cobalancer corresponding to $b_{n}$. What we are going to prove now is much stronger than Theorem 6.1 . 
Theorem 6.2. For $n=1,2, \ldots, R_{n}=b_{n}$ and $r_{n+1}=B_{n}$.

Proof. We know that if $B$ is a balancing number with balancer, $R$ then

$$
R=\frac{-(2 B+1)+\sqrt{8 B^{2}+1}}{2} \quad(\text { see }[1, \text { page } 98]) .
$$

Thus

$$
\begin{aligned}
& R_{n+1}=\frac{-\left(2 B_{n+1}+1\right)+\sqrt{8 B_{n+1}^{2}+1}}{2}, \\
& R_{n-1}=\frac{-\left(2 B_{n-1}+1\right)+\sqrt{8 B_{n-1}^{2}+1}}{2} .
\end{aligned}
$$

Also, from Theorem 3.1 and [1, Corollary 3.2], we have

$$
\begin{aligned}
& B_{n+1}=3 B_{n}+\sqrt{8 B_{n}^{2}+1}, \\
& B_{n-1}=3 B_{n}-\sqrt{8 B_{n}^{2}+1} .
\end{aligned}
$$

Substituting (6.8) and (6.9) into (6.6) and (6.7), respectively, we obtain

$$
\begin{aligned}
R_{n+1} & =\frac{2 B_{n}+\sqrt{8 B_{n}^{2}+1}-1}{2}, \\
R_{n-1} & =\frac{-14 B_{n}+5 \sqrt{8 B_{n}^{2}+1}-1}{2} .
\end{aligned}
$$

Adding the last two equations, we get

$$
\begin{aligned}
R_{n+1}+R_{n-1} & =\frac{-12 B_{n}+6 \sqrt{8 B_{n}^{2}+1}-2}{2} \\
& =6 \cdot \frac{-\left(2 B_{n}+1\right)+\sqrt{8 B_{n}^{2}+1}}{2}+2=6 R_{n}+2 .
\end{aligned}
$$

This gives

$$
R_{n+1}=6 R_{n}-R_{n-1}+2
$$

Thus $R_{n}$ satisfies the same recurrence relation as that of $b_{n}$. Further, since $R_{1}=b_{1}=0$ and $R_{2}=b_{2}=2$, it follows that $R_{n}=b_{n}$ for $n=1,2, \ldots$. This proves the first part of the theorem.

We prove the second part of the theorem in a similar way. Using (1.4), we obtain

$$
\begin{aligned}
& r_{n+1}=\frac{-\left(2 b_{n+1}+1\right)+\sqrt{8 b_{n+1}^{2}+8 b_{n+1}+1}}{2}, \\
& r_{n-1}=\frac{-\left(2 b_{n-1}+1\right)+\sqrt{8 b_{n-1}^{2}+8 b_{n-1}+1}}{2} .
\end{aligned}
$$


Substituting

$$
b_{n+1}=3 b_{n}+\sqrt{8 b_{n+1}^{2}+8 b_{n+1}+1}+1
$$

into (6.13) and

$$
b_{n-1}=3 b_{n}-\sqrt{8 b_{n-1}^{2}+8 b_{n-1}+1}+1
$$

into (6.14), we obtain

$$
\begin{aligned}
r_{n+1} & =\frac{2 b_{n}+\sqrt{8 b_{n+1}^{2}+8 b_{n-1}+1}+1}{2} \\
r_{n-1} & =\frac{-14 b_{n}+5 \sqrt{8 b_{n-1}^{2}+8 b_{n-1}+1}-7}{2} .
\end{aligned}
$$

Adding the last two equations, we get

$$
\begin{aligned}
r_{n+1}+r_{n-1} & =\frac{-12 b_{n}+6 \sqrt{8 b_{n}^{2}+8 b_{n}+1}-6}{2} \\
& =6 \cdot \frac{-\left(2 b_{n}+1\right)+\sqrt{8 b_{n}^{2}+8 b_{n}+1}}{2}=6 r_{n} .
\end{aligned}
$$

Thus $r_{n}$ satisfies the same recurrence relation as that of $B_{n}$. Further, since $B_{1}=r_{2}=1$ and $B_{2}=r_{3}=6$, it follows that $B_{n}=r_{n+1}$ for $n=1,2, \ldots$. This completes the proof of the theorem.

COROLlary 6.3. Every balancer is even.

Proof. The proof follows directly from Theorem 3.1 and Theorem 6.2.

Corollary 6.4. $R_{n+1}=R_{n}+2 B_{n}$.

Proof. The proof follows directly from Corollary 4.2 and Theorem 6.2.

We are now in a position to prove that $h(x)$ and $t(x, y)$ are cobalancing numbers as stated in Theorem 2.1.

We first show that if $x$ is a cobalancing number, then

$$
h(x)=8 x^{2}+8 x+1+(2 x+1) \sqrt{8 x^{2}+8 x+1}+1
$$

is also a cobalancing number.

From [1, Theorem 3.1], we know that if $y$ is a balancing number, then $u=2 y \sqrt{8 y^{2}+1}$ is also a balancing number and the balancer corresponding to $u$ is

$$
R=\frac{-(2 u+1)+\sqrt{8 u^{2}+1}}{2}=8 y^{2}-2 y \sqrt{8 y^{2}+1}
$$


If $x$ is the balancer corresponding to the balancing number $y$, then from (6.3), we find

$$
y=\frac{(2 x+1)+\sqrt{8 x^{2}+8 x+1}}{2}
$$

so that

$$
\begin{aligned}
8 y^{2}+1 & =24 x^{2}+24 x+4(2 x+1) \sqrt{8 x^{2}+8 x+1}+5 \\
& =\left(2(2 x+1)+\sqrt{8 x^{2}+8 x+1}\right)^{2} .
\end{aligned}
$$

Substitution of (6.22) into (6.20) gives

$$
\begin{aligned}
R= & 24 x^{2}+24 x+4(2 x+1) \sqrt{8 x^{2}+8 x+1}+4 \\
& -2\left[\frac{2(2 x+1)+\sqrt{8 x^{2}+8 x+1}}{2}\right] \cdot\left[2(2 x+1)+\sqrt{8 x^{2}+8 x+1}\right] \\
= & 8 x^{2}+8 x+1+(2 x+1) \sqrt{8 x^{2}+8 x+1}=h(x) .
\end{aligned}
$$

Thus for any balancer $x, h(x)$ is always a balancer. Since by Theorem 6.1 every balancer is a cobalancing number, the result follows.

We next prove that if $x$ and $y$ are cobalancing numbers, then

$$
\begin{aligned}
t(x, y)=\frac{1}{2}[ & 2(2 x+1)(2 y+1)+(2 x+1) \sqrt{8 y^{2}+8 y+1} \\
& \left.+(2 y+1) \sqrt{8 x^{2}+8 x+1}+\sqrt{8 x^{2}+8 x+1} \sqrt{8 y^{2}+8 y+1}-1\right]
\end{aligned}
$$

is also a cobalancing number. From [1, Theorem 4.1], we know that if $u$ and $v$ are balancing numbers, then

$$
w=u \sqrt{8 v^{2}+1}+v \sqrt{8 u^{2}+1}
$$

is also a balancing number. Let $s, x$, and $y$ be the balancers corresponding to the balancing numbers $w, u$, and $v$, respectively. Then,

$$
\begin{aligned}
s & =\frac{-(2 w+1)+\sqrt{8 w^{2}+1}}{2} \\
& =\frac{1}{2}\left[8 u v+\sqrt{\left(8 u^{2}+1\right)\left(8 v^{2}+1\right)}-2 u \sqrt{8 v^{2}+1}-2 v \sqrt{8 u^{2}+1}-1\right] .
\end{aligned}
$$

Now substituting

$$
\begin{aligned}
& u=\frac{(2 x+1)+\sqrt{8 x^{2}+8 x+1}}{2}, \\
& v=\frac{(2 y+1)+\sqrt{8 y^{2}+8 y+1}}{2}
\end{aligned}
$$


into (6.26), we find that

$$
\begin{aligned}
s=\frac{1}{2}[ & {\left[2(2 x+1)(2 y+1)+(2 x+1) \sqrt{8 y^{2}+8 y+1}\right.} \\
& \left.+(2 y+1) \sqrt{8 x^{2}+8 x+1}+\sqrt{8 x^{2}+8 x+1} \sqrt{8 y^{2}+8 y+1}-1\right]=t(x, y) .
\end{aligned}
$$

Again since every balancer is a cobalancing number by Theorem 6.1, the result follows.

Remark 6.5. $t(x, x)=h(x)$.

\section{An application of cobalancing numbers to the Diophantine equation $x^{2}+(x+1)^{2}=y^{2}$}

As we know, the Diophantine equation (see [2, page 165]) $x^{2}+(x+1)^{2}=y^{2}, x, y \in \mathbb{Z}^{+}$, is a particular case of the equation $x^{2}+y^{2}=z^{2}, x, y, z \in \mathbb{Z}^{+}$. Any solution $(x, y, z)$ of the later equation is called a Pythagorean triplet. Behera and Panda [1] established a link between the solutions of the equation $x^{2}+(x+1)^{2}=y^{2}$ and balancing numbers. Here we are going to obtain an easy relation between the solutions of this equation with cobalancing numbers.

Let $b$ be any cobalancing number and $r$ its cobalancer and $c=b+r$. Then (1.2) can be rewritten as

$$
1+2+\cdots+b=(b+1)+(b+2)+\cdots+c
$$

from which we find $b$ in terms of $c$ as

$$
b=\frac{-1+\sqrt{2 c^{2}+2 c+1}}{2} .
$$

Thus $2 c^{2}+2 c+1$ is a perfect square, and also

$$
2 c^{2}+2 c+1=c^{2}+(c+1)^{2} .
$$

This suggests that the Diophantine equation $x^{2}+(x+1)^{2}=y^{2}$ has the solution

$$
x=b+r, \quad y=\sqrt{2 c^{2}+2 c+1} .
$$

Take for example $b=14$ so that $r=6$ and $c=b+r=20$. Further, $2 c^{2}+2 c+1=841=29^{2}$ and we have

$$
20^{2}+21^{2}=29^{2}
$$

Similarly for $b=84$, we have $119^{2}+120^{2}=169^{2}$.

\section{Acknowledgment}

It is a pleasure to thank the anonymous referees for their valuable comments and suggestions that greatly improved the presentation of the paper. 


\section{Cobalancing numbers}

\section{References}

[1] A. Behera and G. K. Panda, On the square roots of triangular numbers, Fibonacci Quart. 37 (1999), no. 2, 98-105.

[2] L. E. Dickson, History of the Theory of Numbers. Vol. II-Diophantine Analysis, Chelsea Publishing Company, New York, 1952.

[3] V. Krishnamurthy, Combinatorics: Theory and Applications, Affiliated East-West Press, New Delhi, 1985.

[4] K. B. Subramaniam, A simple computation of square triangular numbers, Internat. J. Math. Ed. Sci. Tech. 23 (1992), no. 5, 790-793.

[5] - A divisibility property of square triangular numbers, Internat. J. Math. Ed. Sci. Tech. 26 (1995), no. 2, 284-286.

[6] _ Almost square triangular numbers, Fibonacci Quart. 37 (1999), no. 3, 194-197.

G. K. Panda: Department of Mathematics, National Institute of Technology, Rourkela-769 008, Orissa, India

E-mail address: gkpanda@nitrkl.ac.in

P. K. Ray: Department of Mathematics, College of Arts, Science and Technology, Bondamunda, Rourkela-770 032, Orissa, India 


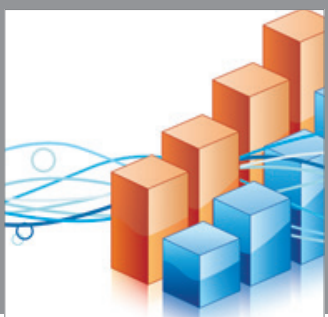

Advances in

Operations Research

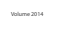

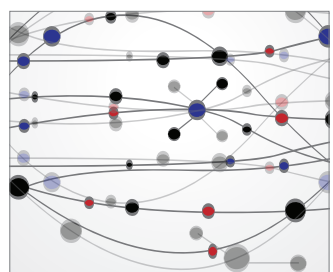

\section{The Scientific} World Journal
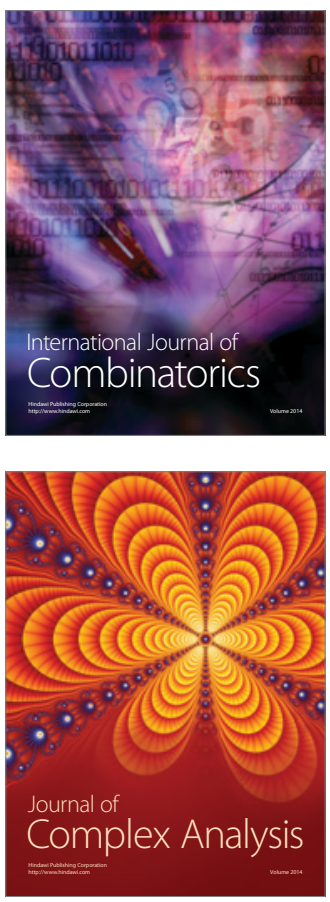

International Journal of

Mathematics and

Mathematical

Sciences
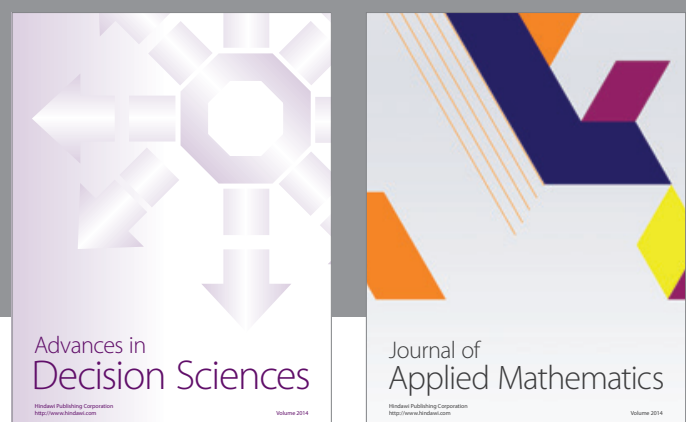

Journal of

Applied Mathematics
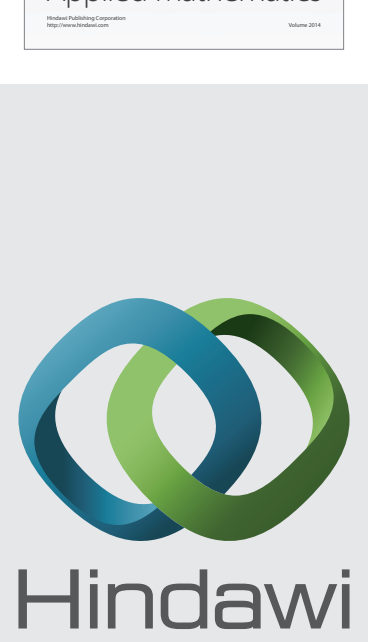

Submit your manuscripts at http://www.hindawi.com
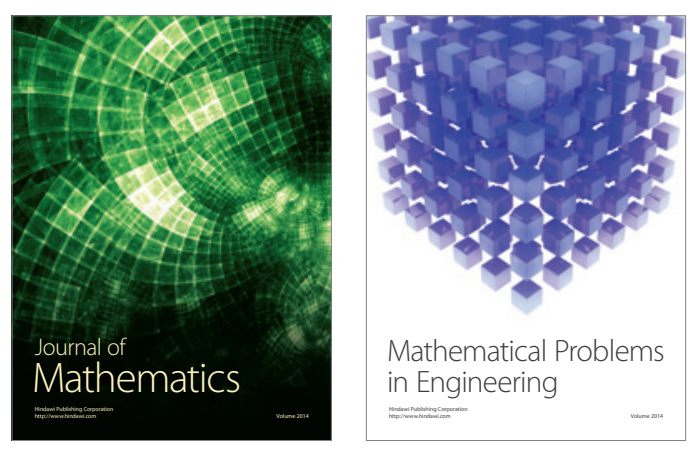

Mathematical Problems in Engineering
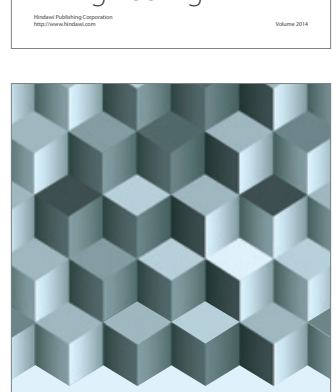

Journal of

Function Spaces
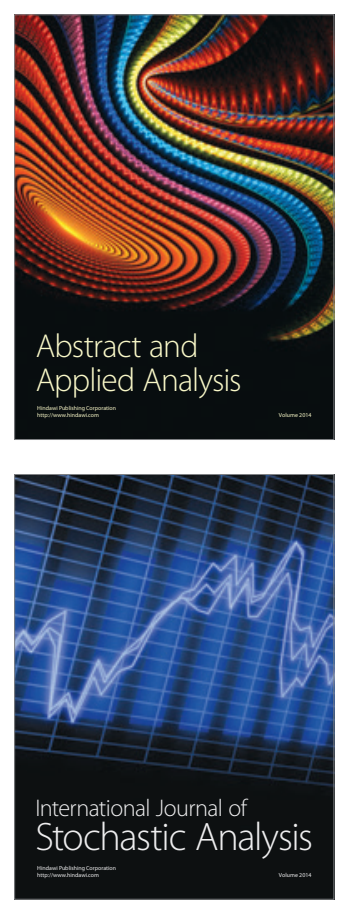

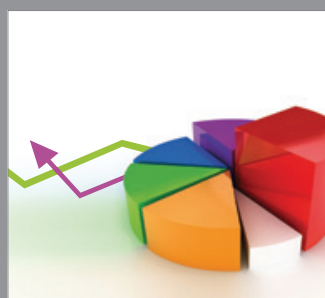

ournal of

Probability and Statistics

Promensencen
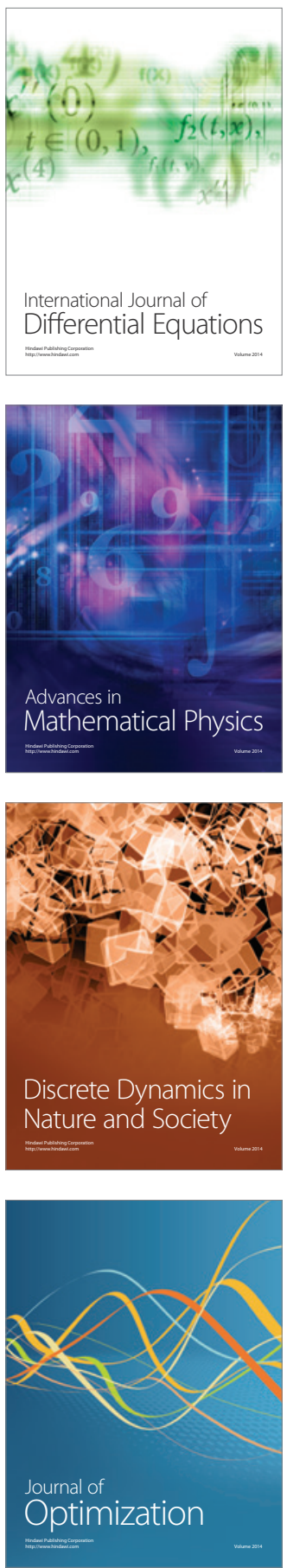